\title{
Design of Constrained Causal Stable IIR Filters Using a New Second- Order-Cone-Programming-Based Model-Reduction Technique
}

\author{
S. C. Chan, Member, IEEE, K. M. Tsui, and K. W. Tse
}

\begin{abstract}
This brief proposes a new method for designing infinite-impulse response (IIR) filter with peak error and prescribed flatness constraints. It is based on the model reduction of a finite-impulse response function that satisfies the specification by extending a method previously proposed by Brandenstein et al. The proposed model-reduction method retains the denominator of the conventional techniques and formulates the optimal design of the numerator as a second-order cone programming problem. Therefore, linear and convex quadratic inequalities such as peak error constraints and prescribed number of zeros at the stopband for IIR filters can be imposed and solved optimally. Moreover, a method is proposed to express the denominator of the model-reduced IIR filter as a polynomial in integer power of $z$, which efficiently facilitates its polyphase implementation in multirate applications. Design examples show that the proposed method gives better performance, and more flexibility in incorporating a wide variety of constraints than conventional methods.
\end{abstract}

Index Terms-Causal stable infinite-impulse response (IIR) filters, linear and convex quadratic constraints, model reduction, polyphase decomposition, second-order cone programming.

\section{INTRODUCTION}

$\mathbf{T}$ HE design of approximately linear-phase infinite-impulse response (IIR) filters is traditionally being considered as a very difficult problem, because the performance measures such as the least-squares (LS) or minimax errors, and the stability constraint are highly nonlinear functions of the filter coefficients. It usually involves constrained nonlinear optimization, whose performance is rather sensitive to the initial guess. Very often, the optimization software will converge to unsatisfactory local minimum if the initial guess is inappropriately chosen. For IIR filters with a high order of denominator, direct optimization and the eigenvalue-based method [1] might find difficulties in ensuring convergence and stability. Therefore, most methods focused on these two issues by some means of problem relaxation (see [2] and references therein). More recently, filter sharpening technique was proposed in [3], but there is no control in the response of the IIR filter so designed. Another indirect but useful method for designing IIR filters is based on model reduction [4], [5] of FIR prototype filters, which can

Manuscript received February 24, 2005; revised January 17, 2006, and May 13, 2006. This paper was presented in part at 12th European Signal Processing Conference (EUSIPCO'04), Vienna, Austria, September 6-10, 2004. This paper was recommended by Associate Editor Y.-P. Lin.

The authors are with the Department of Electrical and Electronic Engineering, The University of Hong Kong, Hong Kong (e-mail: scchan@eee.hku.hk; kmtsui@eee.hku.hk; kwtse@eee.hku.hk).

Digital Object Identifier 10.1109/TCSII.2006.883831 be designed easily and optimally using existing methods such as the Remez exchange algorithm, semidefinite programming [6] and second-order cone programming (SOCP) [7], [8]. Basically, a finite-impulse response (FIR) prototype filter with the given specifications is first designed. Model reduction is then applied to convert this FIR filter to an IIR filter of reduced order. In addition to its simple design procedure, the advantage of the model-reduction approach is that the resulting IIR filter is guaranteed to be stable, and the frequency characteristics such as the phase response of the FIR prototype filter is well preserved. However, it does not allow precise control of the frequency response and incorporation of other constraints [5], such as prescribed number of zeros at the stopband or peak ripple constraints. One would also expect the performance of the filter to be sub-optimal and it can be further improved.

In this brief, we propose a new design method for IIR filters using a new constrained model-reduction technique, which is a modification of the model-reduction method proposed in [4]. Important advantages of the method in [4] are that the numerator and denominator can be determined separately and the stability of the model-reduced filter is guaranteed. This property allows us to incorporate linear and convex quadratic constraints and improve the frequency response of the final IIR filter by designing the numerator using SOCP, given the denominator at the first stage. For illustrative purposes, we mainly focus on the incorporation of peak stopband error and prescribed number of zeros in the stopband to the final IIR filters. The former is useful to limit the undesirable sidelobes at the band edges and design results show that it yields considerable better performance compared to conventional model-reduction methods. Owing to the improved frequency characteristics of the proposed design method, further optimization is usually not required. Also, we propose a modification of the new model-reduction method so that the denominator of the model-reduced filter is a polynomial in $z^{M}$, where $M$ is an integer. This is very useful in expressing the model-reduced filters in its polyphase representation, which provides efficient implementation of decimation/interpolation filters and other multirate applications such as discrete Fourier transform (DFT) filter banks (FBs) [9], [10]. Using these results, the design approach is further extended to the design of low-delay IIR DFT FBs with much lower arithmetic complexity than the approach in [10] because of the better utilization of the polyphase structure. The resulting low-delay IIR DFT FBs are thus well suited to applications requiring lower system delays. They also offer comparable implementation complexity and performance as conventional linear-phase DFT FBs.

The brief is organized as follows. The principle of the proposed constrained model reduction is described in Section II. 
The details of the SOCP formulation of the peak error and magnitude flatness constraints for IIR filters are given in Section III. Also, the method to express the denominator of the model-reduced filter as a polynomial in $z^{M}$ is proposed. Design examples, including the design of a low-delay IIR DFT FB, are given in Section IV to demonstrate the effectiveness of the proposed approach. Finally, conclusions are drawn in Section V.

\section{Proposed Model-ReduCtion METHOD}

Suppose that we have designed a FIR filter of length $L$ $H(z)=\sum_{n=0}^{L-1} h(n) z^{-n}$, using any existing methods in the literature to satisfy a given specification. The idea of model reduction is to approximate $H(z)$ by an IIR filter $\hat{H}(z)$ with the following form:

$$
\hat{H}(z)=\frac{P(z)}{Q(z)}=\frac{\sum_{n=0}^{L_{p}-1} p(n) z^{-n}}{\sum_{n=0}^{L_{q}-1} q(n) z^{-n}}, \quad q(0)=1, \quad L_{p} \geq L_{q}
$$

where $L_{p}$ and $L_{q}$, are, respectively, the length of the numerator and denominator of $\hat{H}(z)$. As mentioned earlier, the advantage of the method in [4] is that $Q(z)$ can be found without the knowledge of $P(z)$, unlike other model-reduction techniques. Based on this observation, we propose to design $P(z)$ using SOCP, where additional constraints can be incorporated to improve the performance during the determination of $P(z)$, after $Q(z)$ has been found. We now describe the procedures for determining $Q(z)$ and $P(z)$.

\section{A. Determination of Denominator}

In [4], $Q(z)$ is determined using a simple iterative procedure. Let us denote the polynomial which approximates $Q(z)$ at the $k$ th iteration as $Q^{(k)}(z)=1+\sum_{n=1}^{L_{q}-1} q^{(k)}(n) z^{-n},\left(Q^{(0)}(z)=\right.$ 1). By defining $X^{(k)}(z)=z^{-(\bar{L}-1)} H\left(z^{-1}\right) / Q^{(k-1)}(z)=$ $\sum_{n=0}^{\infty} x^{(k)}(n) z^{-n}, q^{(k)}(n)$ can be obtained by minimizing

$$
F^{(k)}=\left(\boldsymbol{B}^{(k)} \boldsymbol{q}^{(k)}-\boldsymbol{d}^{(k)}\right)^{T}\left(\boldsymbol{B}^{(k)} \boldsymbol{q}^{(k)}-\boldsymbol{d}^{(k)}\right)
$$

where

$$
\begin{aligned}
& \boldsymbol{B}^{(k)}=\left[\begin{array}{cccc}
\boldsymbol{x}^{(k)}(0) & 0 & \ldots & 0 \\
\boldsymbol{x}^{(k)}(1) & \boldsymbol{x}^{(k)}(0) & \ddots & \vdots \\
\vdots & \vdots & \ddots & 0 \\
\boldsymbol{x}^{(k)}\left(L_{q}-2\right) & \boldsymbol{x}^{(k)}\left(L_{q}-3\right) & \cdots & \boldsymbol{x}^{(k)}(0) \\
\vdots & \vdots & & \vdots \\
\boldsymbol{x}^{(k)}(L-2) & \boldsymbol{x}^{(k)}(L-3) & \cdots & \boldsymbol{x}^{(k)}\left(L-L_{q}\right)
\end{array}\right] \\
& \boldsymbol{q}^{(k)}=\left[\begin{array}{ccc}
\left.q^{(k)}\left(L_{q}-1\right), \ldots, q^{(k)}(1)\right]^{T} & \text { and }
\end{array}\right. \\
& \boldsymbol{d}^{(k)}=-\left[0, \ldots, 0, \boldsymbol{x}^{(k)}(0), \ldots, \boldsymbol{x}^{(k)}\left(L-L_{q}-1\right)\right]^{T} .
\end{aligned}
$$

The basic idea is to find $\boldsymbol{q}^{(k)}$ such that $F^{(k)}$ is the smallest among all the iterations for a sufficiently large $k$. More importantly, the roots of the resulting $Q^{(k)}(z)$, which minimizes $F^{(k)}$ ( for an arbitrarily given $X^{(k)}(z)$, are proved to lie strictly inside the unit circle, and thus $\hat{H}(z)$ is always stable. Interested readers are referred to [4] for more details.

\section{B. Determination of Numerator Using SOCP}

Once $Q\left(e^{j \omega}\right)$ is designed, $\hat{H}\left(e^{j \omega}\right)$ in (2-1) can be rewritten more compactly in matrix form of the design variables $\boldsymbol{p}=$ $\left[p(0), \ldots, p\left(L_{p}-1\right)\right]^{T}$ as follows:

$$
\hat{H}\left(e^{j \omega}\right)=\boldsymbol{p}^{T}[\boldsymbol{c}(\omega)+j \boldsymbol{s}(\omega)]
$$

where $\boldsymbol{c}(\omega)=\operatorname{Re}\left[\boldsymbol{e}(\omega) / Q\left(e^{j \omega}\right)\right], \boldsymbol{s}(\omega)=\operatorname{Im}\left[\boldsymbol{e}(\omega) / Q\left(e^{j \omega}\right)\right]$; $\boldsymbol{e}(\omega)=\left[1, e^{-j \omega} \ldots, e^{-j\left(L_{p}-1\right) \omega}\right]^{T}$, and $\operatorname{Re}[x]$ and $\operatorname{Im}[x] \mathrm{de}-$ note, respectively, the real and imaginary parts of $x$. Here, we want to approximate the response of $H(z)$ by $P(z)$, given $Q(z)$, in the LS sense. That is

$$
\begin{aligned}
E_{L S}(\boldsymbol{p}) & =\int_{-\pi}^{\pi}\left|\left\{P\left(e^{j \omega}\right) / Q\left(e^{j \omega}\right)\right\}-H\left(e^{j \omega}\right)\right|^{2} d \omega \\
& =\boldsymbol{p}^{T} \boldsymbol{U} \boldsymbol{p}-2 \boldsymbol{p}^{T} \boldsymbol{g}+a
\end{aligned}
$$

where $\boldsymbol{U}=\int_{-\pi}^{\pi}\left[\boldsymbol{c}(\omega) \boldsymbol{c}(\omega)^{T}+\boldsymbol{s}(\omega) \boldsymbol{s}(\omega)^{T}\right] d \omega, \quad g=$ $\int_{-\pi}^{\pi}\left\{\boldsymbol{c}(\omega) \operatorname{Re}\left[H\left(e^{j \omega}\right)\right]+\boldsymbol{s}(\omega) \operatorname{Im}\left[H\left(e^{j \omega}\right)\right]\right\} d \omega$, and $a=$ $-\pi$ $\int_{-\pi}^{\pi}\left|H\left(e^{j \omega}\right)\right|^{2} d \omega$. This is a standard quadratic programming problem, where the optimal solution is given by $\boldsymbol{p}_{L S}=\boldsymbol{U}^{-1} \boldsymbol{g}$. However, large sidelodes are usually encountered at the band-edge of the model-reduced filter. In order to improve its frequency characteristic, we propose to minimize $E_{L S}(\boldsymbol{p})$ using SOCP so that additional constraints on the stopband ripple constraints can be incorporated. More specifically, (2-4) is first rewritten as $E_{L S}(\boldsymbol{p})=\left\|\boldsymbol{U}^{1 / 2} \boldsymbol{p}-\boldsymbol{U}^{-1 / 2} \boldsymbol{g}\right\|^{2}-\left(\boldsymbol{g}^{T} \boldsymbol{U}^{-1} \boldsymbol{g}-a\right)$, where $\|\bullet\|$ denotes the Euclidean norm. By defining the augmented variable $\boldsymbol{x}=\left[\delta \boldsymbol{p}^{T}\right]^{T}$, the LS design problem can be reformulated as the following SOCP:

$$
\min _{\boldsymbol{x}} \boldsymbol{c}^{T} \boldsymbol{x} \quad \text { subject to } \boldsymbol{c}^{T} \boldsymbol{x} \geq\|\overline{\boldsymbol{U}} \boldsymbol{x}-\overline{\boldsymbol{g}}\|
$$

where $\boldsymbol{c}=\left[1 \boldsymbol{O}_{L_{p}}^{T}\right]^{T}, \overline{\boldsymbol{U}}=\left[\boldsymbol{O}_{L_{p}} \boldsymbol{U}^{1 / 2}\right], \overline{\boldsymbol{g}}=\boldsymbol{U}^{-1 / 2} \boldsymbol{g}$, and $\boldsymbol{O}_{N}$ is a $N$ row zero vector. Since SOCP is a convex problem, the optimal solution, if it exists, can be found. Another advantage is that additional linear equalities and convex quadratic constraints can also be formulated as SOCP, as we shall illustrate later.

\section{Selection of the Length of Denominator}

Suppose that the desired passband response of $H(z)$, denoted by $H_{p}(z)$, is given by $H_{p}\left(e^{j \omega}\right)=$ $e^{-j \omega \tau}, \quad \omega$ in the passband, where $\tau=(L-1) / 2-D$ is the passband group delay of $H(z)$, and $D$ is a prescribed delay reduction parameter. $D=0$ corresponds to its linear-phase counterpart. In order to approximate $H(z)$ to a sufficiently small error level for the technique in [4], we found that the length of the denominator of $\hat{H}(z)$ should satisfy $L_{q} \geq\lceil\tau\rceil+1$, where $\lceil w\rceil$ denotes the integer just larger than or equal to $w$. This tells us that the savings in number of multiplications and additions would be more pronounced if model reduction is applied to FIR functions with lower system delay. Here, we prefer to choose $D \cong L / 4$ such that $\tau \cong L / 4$. It is because a bump will usually appear at the transition band when the 
group delay is lower than this value. This choice allows us to set the system delay of $H(z)$ as low as possible, while keeping a good frequency characteristic of $H(z)$. Therefore, the implementation complexity and performance of $\hat{H}(z)$ would be comparable to its linear phase FIR counterpart. However, the system delay is approximately reduced by a factor of two.

\section{DESIGN OF CONSTRAINED IIR FILTER}

\section{A. Peak Stopband Error Constraint}

Let $\varepsilon$ be the prescribed peak stopband ripple to be imposed on the model-reduced filter, $\hat{H}(z)$, i.e., $\left|\hat{H}\left(e^{j \omega}\right)\right| \leq \varepsilon$ in the stopband. Using (2-1) with given $Q(z)$, the constraint can be rewritten as follows:

$$
\varepsilon \geq\|\boldsymbol{R} \boldsymbol{x}\|, \quad \boldsymbol{R}=\left[\begin{array}{ll}
0, & \boldsymbol{c}(\omega)^{T} \\
0, & \boldsymbol{s}(\omega)^{T}
\end{array}\right] .
$$

Discretizing (3-1), these constraints on the peak ripples can be augmented to the existing SOCP in (2-5) for determining $P(z)$.

\section{B. Imposing Linear Equality Constraint}

To impose $U_{\bar{\omega}}$ zeros on $\hat{H}(z)$ at $\omega=\bar{\omega}$ in the stopband, the following relation should be satisfied:

$$
\left.\frac{d^{u}}{d z^{u}} \hat{H}(\omega)\right|_{\omega=\bar{\omega}}=0, \quad u=0,1, \ldots, U_{\bar{\omega}}-1 .
$$

which is also equivalent to

$$
\left.\frac{d^{u}}{d z^{u}} P(\omega)\right|_{\omega=\bar{\omega}}=0, \quad u=0,1, \ldots, U_{\bar{\omega}}-1 .
$$

Expanding (3-3) and after slight manipulation, one gets a set of linear equality constraints as follows:

$$
\sum_{n=0}^{L_{p}-1} n^{u} e^{-j \bar{\omega} n} p(n)=0, \quad u=0,1, \ldots, U_{\bar{\omega}}-1
$$

and its matrix form is given by $\boldsymbol{A} \cdot \boldsymbol{p}=\boldsymbol{b}$, where $[\boldsymbol{A}]_{u, n}=$ $n^{u} e^{-j \bar{\omega} n}$ and $[\boldsymbol{b}]_{u}=0$. Here, $[\boldsymbol{A}]_{m, n}$ denotes the $(m, n)$ th entry of the $\left(U_{\bar{\omega}} \times L_{p}\right)$ matrix $\boldsymbol{A}$. The problems in (2-5) can then be solved subject to these linear equality constraints using SOCP.

\section{Polyphase Decomposition}

Polyphase representation of digital filters is useful to the efficient implementation of decimation/interpolation filters, as well as other multirate systems [9]. However, expressing the IIR filters with the form of (2-1) in its polyphase representation is rather inefficient. In the conventional method, the rational function in (2-1) is rewritten as follows [10]:

$$
\begin{aligned}
\hat{H}(z) & =\frac{\left(\sum_{n=0}^{L_{p}-1} p(n) z^{-n}\right)\left(\prod_{k=1}^{L_{q}-1}\left(\sum_{m=0}^{M-1} \lambda_{k}^{m} z^{-m}\right)\right)}{\prod_{k=1}^{L_{q}-1}\left(1-\lambda_{k}^{M} z^{-M}\right)} \\
& =\frac{\widetilde{P}(z)}{\widetilde{Q}\left(z^{M}\right)}
\end{aligned}
$$

where $\lambda_{k}$ 's are the roots of $Q(z)$ and $M$ is a positive integer. It is noticed that the lengths of $\widetilde{P}(z)$ and $\widetilde{Q}(z)$ respectively increases considerably to $M\left(L_{q}-1\right)-L_{q}+L_{p}+1$ and $M\left(L_{q}-1\right)+1$. The $M$ th polyphase decomposition of (3-5) is then given by $\hat{H}(z)=$ $\sum_{m=0}^{M-1} z^{-m} E_{m}\left(z^{M}\right)$, where $E_{m}(z)=E_{\widetilde{P}, m}(z) / \widetilde{Q}(z)$ and $E_{\widetilde{P}, m}(z)$ are the $m$ th polyphase components of $\hat{H}(z)$ and $\widetilde{P}(z)$, respectively. One drawback of such decomposition is the potential numerical errors in estimating the roots of $Q(z)$, especially when $L_{q}$ is large. Moreover, the lengths of $\widetilde{P}(z)$ and $\widetilde{Q}(z)$ are unnecessarily large when $M$ is large.

Therefore, we propose to generate a denominator polynomial in $z^{M}$ directly from model reduction. To this end, the design procedure of determining the denominator, as described in Section II-A, is modified. Instead of using the rational function as in (2-1), the model-reduced filter is assumed to have the following form:

$$
\begin{aligned}
\hat{H}(z) & =\frac{P(z)}{Q\left(z^{M}\right)} \frac{\sum_{n=0}^{L_{p}-1} p(n) z^{-n}}{\sum_{n=0}^{d} q(n) z^{-n M}} \\
q(0) & =1, \quad L_{p} \geq d M+1
\end{aligned}
$$

where $d$ corresponds to the number of nonzero coefficients of $Q\left(z^{M}\right)$, excluding $q(0)$. The vector $\boldsymbol{q}^{(k)}$ in (2-2) is modified as

$$
\boldsymbol{q}^{(k)}=\left[q^{(k)}(d M), q^{(k)}((d-1) M), \ldots, q^{(k)}(M)\right]^{T}
$$

which can be solved by considering the corresponding rows of $\boldsymbol{B}^{(k)}$ and $\boldsymbol{d}^{(k)}$. According to [4], this modification does not violate the stability theorem, which holds for arbitrarily given $X^{(k)}(z)$. Hence, the IIR filter in the form of (3-6) is still stable, provided that $F^{(k)}$ is the smallest. Moreover, it simplifies considerably the procedures of polyphase decomposition, as compared with their counterparts in the form of (2-1). Note that the reciprocal of denominator of IIR filter in this form can be viewed as the equalizer of the interpolated FIR filter proposed by Cabezas and Diniz [11], which is particularly useful in reducing the implementation complexity of FIR filters with very sharp cutoff. The design result presented in Section IV also shows that the filter so obtained has a considerably lower order than that using the direct decomposition described above.

\section{DESIGN EXAMPLES}

Example 1: Low-Delay IIR Low-Pass Filters: In this example, low-delay IIR low-pass filters are designed using the proposed constrained model-reduction method. All the SOCP problems were solved by the SeDuMi Matlab Toolbox [12] in a Pentium 4 personal computer. The passband and stopband cutoff frequencies are, respectively, $\omega_{p}=0.125 \pi$ and $\omega_{s}=0.15 \pi$. A low-delay FIR prototype filter of length $L=179$ and delay-reduction parameter $D=45$ is first designed using SOCP [8] so that its passband group delay is about 44 samples. The model reduction in [4] is then applied to convert this prototype filter to an IIR filter. According to Section II-C, the lengths of the numerator and denominator of the model-reduced filter are chosen as $L_{p}=45$ and $L_{q}=45$, respectively. Model reducing this prototype filter without any constraints gives the worst case passband deviation of $0.079 \mathrm{~dB}$ 

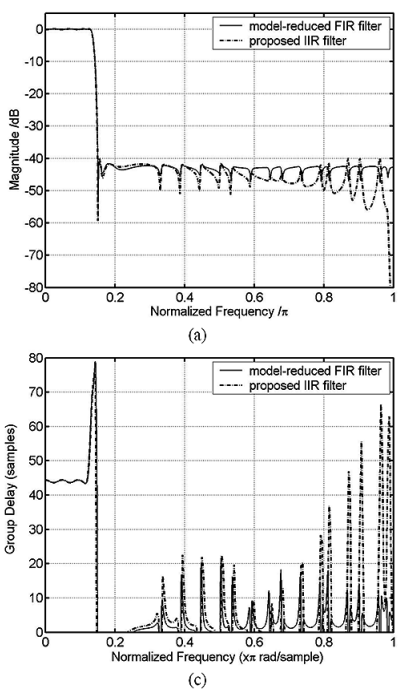
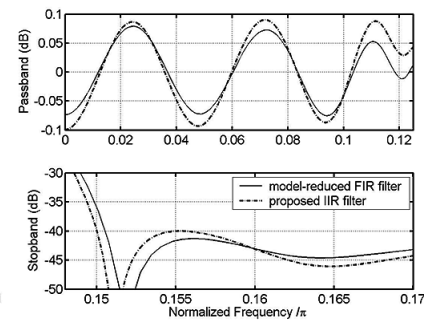

(b)

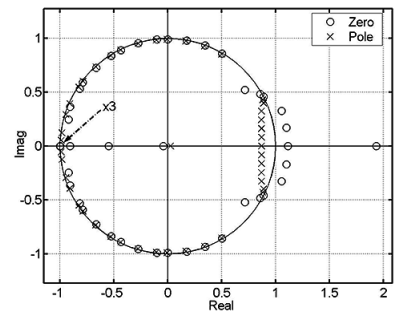

(d)
Fig. 1. Design results of low-delay IIR filters in example 1 (peak stopband error constraint $\varepsilon=40 \mathrm{~dB}$ and three zeros at $\omega=\pi$ ). (a) Frequency response. (b) Passband (top) and stopband (bottom) details. (c) Group delay response of low-pass IIR filters: solid line-model-reduced FIR filter; dash-dotted line-proposed IIR filter. (d) Pole-zero plot of proposed IIR filter.

and the worst case stopband attenuation of $36 \mathrm{~dB}$, as shown by the solid line in Fig. 1(a) and 1(b). As a comparison, we have also implemented the conventional model-reduction technique in [5] for this unconstrained case. The result is similar to that obtained by the approach proposed in [5]. For simplicity, details are omitted.

To limit the sidelobe at the band edge, a peak stopband error constraint of $40 \mathrm{~dB}$ is imposed to the model-reduced filter. In addition, three zeros at $\omega=\pi$ are also imposed. Fig. 1 shows the design results of the IIR filter so obtained. It can be seen from the dash-dotted line in Fig. 1(a) and 1(b) that the worst case stopband attenuation of the proposed IIR filter is now increased to $40 \mathrm{~dB}$ at the expense of slightly lower performance at the passband. Also, as depicted in Fig. 1(d), three zeros at $\omega=\pi$ are successfully imposed by the proposed method to the IIR filter.

Regarding the arithmetic complexity, the implementation of the proposed IIR filter requires 89 multipliers and 87 adders, which is about half of those required for a low-delay prototype FIR filter, and are comparable to those of a length-161 linear-phase FIR filter for a similar performance. However, the linear-phase FIR filter has a passband group delay of 80 samples, which is about two times larger than that of the proposed IIR filter. This demonstrates the effectiveness of the proposed method in designing high-order IIR filter.

Example 2: Low-Delay IIR DFT FB: An $M$-channel uniform DFT FB consists of a set of bandpass filters $h_{m}[n]$, equally spaced at center frequencies $\omega_{m}=2 m \pi / M, m=1, \ldots, M$, which are obtained by modulating a low-pass prototype filter $h_{o}[n]$ of length $N$ with real coefficients. In [13], DFT FBs were proposed to realize intermediate frequency (IF) channelizers in software radio base station. The basic principle of the DFT FB-based channelizer is to extract different radio channels from the IF spectrum, and down-convert them back to baseband, at a lower sample rate suitable for baseband processing. Since the DFT FB can be efficiently implemented as the polyphase struc

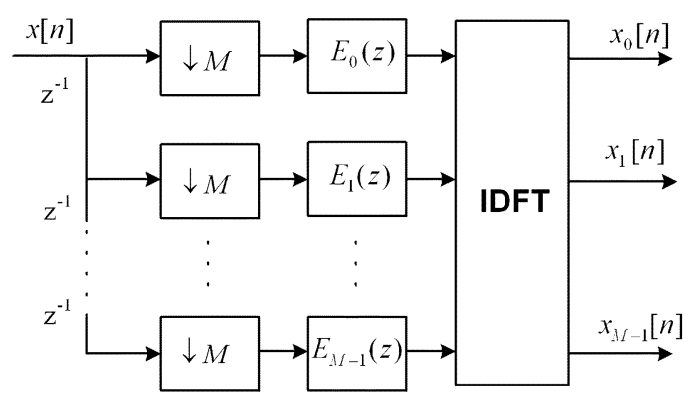

Fig. 2. DFT FB with polyphase decomposition.

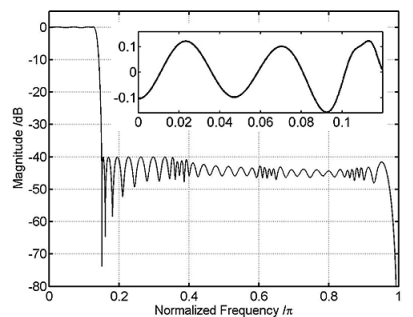

(a)

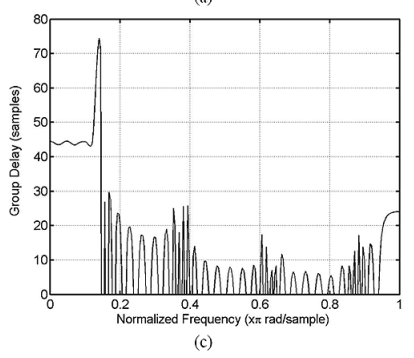

Fig. 3. Design results of low-delay IIR filters in example 2 (peak stopband error constraint $\varepsilon=40 \mathrm{~dB}$ and three zeros at $\omega=\pi$ ). a) Frequency response (passband details in smaller figure), b) impulse response of the numerator (top) and denominator (bottom), c) group delay response, and d) pole-zero plot of proposed IIR filter. The denominator is a polynomial in $z^{8}$.

ture in Fig. 2, its complexity is lower than that of the traditional analog channelizer.

In this example, we shall consider the application of the proposed model-reduction technique to realize a low-delay IIR DFT FB. As an illustration, an eight-channel DFT FB is considered so that the specifications of the low-pass prototype filter are the same as those in example 1. To facilitate the polyphase implementation of the DFT FB in Fig. 2, the IIR filter designed in example 1 has to be expressed in its polyphase representation with $M=8$. Using the decomposition in (3-5), the lengths of its numerator and denominator, however, become 353, and the total number of nonzero coefficients is 398 . On the other hand, the proposed method in Section III-C is also employed with the denominator being constrained to the form of $Q\left(z^{8}\right)$. With $L_{p}=81$ and $L_{q}=81$ (i.e., $d=10$ ), the performances of the IIR filter so obtained are comparable to those of its counterpart in example 1 designed using the proposed method without imposing any constraints. The corresponding worst case passband deviation and stopband attenuation are 0.08 and $36.22 \mathrm{~dB}$, respectively. Moreover, its total number of nonzero coefficients is only 92 , which is about four times less than that of the counterpart. Therefore, its implementation complexity for the polyphase representation is much lower. If the peak stopband error constraint of $40 \mathrm{~dB}$ and three zeros at $\omega=\pi$ are also imposed, design results in Fig. 3 are obtained. It can be seen that all constraints are satisfied. Fig. 4 shows 


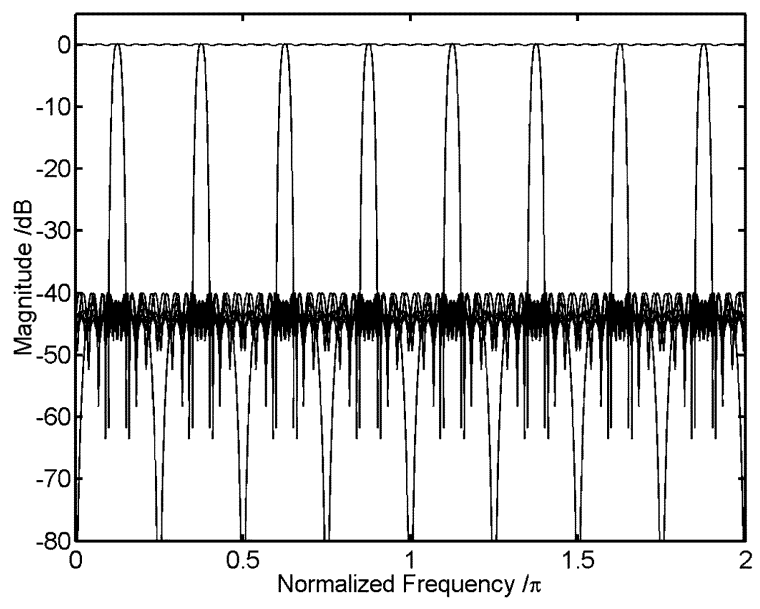

Fig. 4. Frequency response of the low-delay causal stable IIR DFT FB.

the corresponding frequency response of the low-delay IIR DFT FB. The above results illustrate the effectiveness of the proposed model-reduction approach in the efficient design and realization of IIR filters in their polyphase representations.

It should be noted that the number of multiplications per unit time (MPU) of the proposed IIR DFT FB in each polyphase branch is $(81 / 8+10)=20.125$, while that of the low-delay FIR filter is $179 / 8=22.375$. However, if we implement the denominator of the proposed IIR filter at the input followed by the decimator and then the numerators, the actual number of multipliers required will be $(10+81)=91$, which is about half of that for the FIR case. Although the denominator is operating at a higher rate, the denominator is now a polynomial in $z^{M}$, which simplifies considerably the pipelining of the denominator. In fact, this problem was studied in [14], where some of the coefficients in the denominator are set to zero to allow more time for pipeline computation. Moreover, another possible advantage of the proposed IIR DFT FB is that the common denominator of the polyphase components of the proposed IIR filter can be moved to the output of the IDFT. If less than $M$ channels are of interest, the number of MPU of the proposed IIR DFT FB can be further reduced.

\section{CONCLUSION}

A new method for designing IIR filters with peak error and magnitude flatness constraints is proposed. It is based on the model reduction of FIR prototype filters by a new model-reduction technique. The method retains the denominator of the conventional techniques and formulates the optimal design of the numerator as an SOCP problem. Linear and convex quadratic inequalities such as peak error constraints and prescribed number of zeros in the stopband for the IIR filters can be imposed and solved optimally. Moreover, a method to express the denominator of the model-reduced filter as a polynomial in the integer power of $z$ is also proposed to facilitate its polyphase implementation in multirate applications.

\section{REFERENCES}

[1] X. Zhang and H. Iwakura, "Design of IIR digital filters based on eigenvalue problem," IEEE Trans. Signal Process., vol. 44, no. 6, pp. 1325-1333, Jun. 1996.

[2] W.-S. Lu and T. Hinamoto, "Optimal design of IIR digital filters with robust stability using conic-quadratic-programming updates," IEEE Trans. Signal Process., vol. 51, no. 6, pp. 1581-1592, Jun. 2003.

[3] S. Engelberg, "A more general approach to the filter sharpening technique of Kaiser and Hamming," IEEE Trans. Circuits Syst. II, Exp. Briefs, vol. 53, no. 7, pp. 538-540, Jul. 2006.

[4] H. Brandenstein and R. Unbehauen, "Least-squares approximation of FIR by IIR filters," IEEE Trans. Signal Process., vol. 46, no. 1, pp. 21-30, Jan. 1998.

[5] B. C. Moore, "Principal component analysis in linear system: Controllability, observability, and model reduction," IEEE Trans. Autom. Contr., vol. AC-26, no. 1, pp. 17-31, Jan. 1981.

[6] W.-S. Lu and A. Antoniou, "Design of digital filters and filter banks by optimization: A state of the art review," in Proc. Eur. Signal Process. Conf. (EUSIPCO'00), Sep. 2000, vol. 1, pp. 351-354.

[7] J. O. Coleman and D. P. Scholnik, "Design of nonlinear phase FIR filters with second-order cone programming," in Proc. IEEE MWSCAS 1999, Aug. 1999, vol. 1, pp. 409-412.

[8] K. M. Tsui, S. C. Chan, and K. S. Yeung, "Design of FIR digital filters with prescribed flatness and peak error constraints using second-order cone programming," IEEE Trans. Circuits Syst. II, Exp. Briefs, vol. 52, no. 9, pp. 601-605, Sep. 2005.

[9] P. P. Vaidyanathan, Multirate Systems and Filter Banks. Englewood Cliffs, NJ: Prentice-Hall, 1993.

[10] M. G. Bellanger, G. Bonnerot, and M. Coudreuse, "Digital filtering by polyphase network: Application to sample-rate alteration and filter banks," IEEE Trans. Acoust. Speech, Signal Process., vol. ASSP-24, no. 2, pp. 109-114, Apr. 1976.

[11] J. C. E. Cabezas and P. S. R. Diniz, "FIR filters using interpolated prefilters and equalizers," IEEE Trans. Circuits Syst., vol. 37, no. 1, pp. 17-23, Jan. 1990.

[12] J. F. Sturm, "Using SeDuMi 1.02, a MATLAB toolbox for optimization over symmetric cones," Optim. Math. Softw., vol. 11-12, pp. 625-653, 1999.

[13] K. C. Zangi and R. Koilpillai, "Software radio issues in cellular base stations," IEEE J. Select. Areas Commun., vol. 17, no. 4, pp. 561-573, Apr. 1999.

[14] Z. Jiang and A. N. Wilson, Jr., "Design and implementation of efficient pipelined IIR digital filters,' IEEE Trans. Signal Process., vol. 43, no. 3, pp. 579-590, Mar. 1995.

[15] S. C. Chan, K. M. Tsui, and K. W. Tse, "Design of constrained IIR and interpolated IIR filters using a new semi-definite programming based model-reduction technique," in Proc. Eur. Signal Process. Conf. (EUSIPCO’04), Vienna, Austria, Sep. 6-10, 2004, pp. 141-144. 